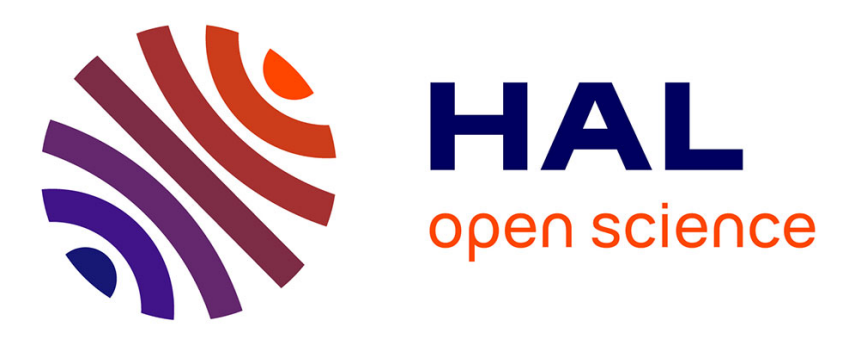

\title{
Jump-to-contact instability: The nanoscale mechanism of droplet coalescence in air
}

\author{
Véronique Incerti, Matthieu Protat, Frédéric Risso, Thierry Ondarçuhu, \\ Philippe Tordjeman
}

\section{- To cite this version:}

Véronique Incerti, Matthieu Protat, Frédéric Risso, Thierry Ondarçuhu, Philippe Tordjeman. Jumpto-contact instability: The nanoscale mechanism of droplet coalescence in air. Physical Review Fluids, 2018, 3 (10), pp.102001. 10.1103/PhysRevFluids.3.102001 . hal-02047629

\author{
HAL Id: hal-02047629 \\ https://hal.science/hal-02047629
}

Submitted on 25 Feb 2019

HAL is a multi-disciplinary open access archive for the deposit and dissemination of scientific research documents, whether they are published or not. The documents may come from teaching and research institutions in France or abroad, or from public or private research centers.
L'archive ouverte pluridisciplinaire HAL, est destinée au dépôt et à la diffusion de documents scientifiques de niveau recherche, publiés ou non, émanant des établissements d'enseignement et de recherche français ou étrangers, des laboratoires publics ou privés. 


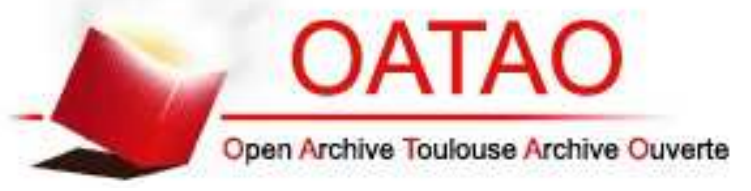

\section{Open Archive Toulouse Archive Ouverte}

OATAO is an open access repository that collects the work of Toulouse researchers and makes it freely available over the web where possible

This is an author's version published in: http://oatao.univ-toulouse.fr/22944

\section{Official URL:}

https://doi.org/10.1103/PhysRevFluids.3.102001

\section{To cite this version:}

Incerti, Véronique and Protat, Matthieu and Risso, Frédéric and Ondarçuhu, Thierry and Tordjeman, Philippe. Jump-tocontact instability: The nanoscale mechanism of droplet coalescence in air. (2018) Physical Review Fluids, 3 (10). 102001. ISSN 2469-990X

Any correspondence concerning this service should be sent to the repository administrator: tech-oatao@listes-diff.inp-toulouse.fr 


\title{
Jump-to-contact instability: The nanoscale mechanism of droplet coalescence in air
}

\author{
Véronique Chireux, Matthieu Protat, Frédéric Risso, Thierry Ondarçuhu, and Philippe Tordjeman \\ Institut de Mécanique des Fluides de Toulouse (IMFT), Université de Toulouse, \\ CNRS, 31400 Toulouse, France
}

(Received 26 July 2018; published 19 October 2018)

\begin{abstract}
We study experimentally by means of atomic force microscopy (AFM) the jump-tocontact instability between two droplets in air, with radii ranging between 0.7 and $74 \mu \mathrm{m}$. This instability which occurs at the nanoscale is responsible for droplet coalescence. The AFM experiments were conducted in contact and frequency-modulation modes where the interaction force and the frequency shift are monitored while the two droplet interfaces approach each other. The critical distance $d_{\min }$ at which the jump to contact takes place is determined by fitting the experimental curves by the theoretical expressions for the force and the frequency shift. The results point out the existence of two regimes. For submicrometer droplets, $d_{\min }$ scales as $\left(H R_{\mathrm{eq}} / \gamma\right)^{1 / 3}$ where $R_{\mathrm{eq}}$ is the equivalent droplet radius, $H$ the Hamaker constant, and $\gamma$ the surface tension of the liquid. For larger droplets, $d_{\min }$ no longer depends on the droplet size and scales as $(H / \gamma)^{1 / 2}$. This second scaling is the one that controls droplet coalescence in most situations.
\end{abstract}

DOI: 10.1103/PhysRevFluids.3.102001

Coalescence of liquid droplets plays a fundamental role in many industrial applications. Liquidliquid extraction, emulsification, polymer blending processes, and multiphase flows are examples of processes encountered in chemical, pharmaceutical, petroleum, and nuclear industries. Cell-cell interactions are also involved in many biological processes. All these processes are based on the mechanisms of coalescence of soft and deformable bodies, which interact with each other, sometimes under flow. Coalescence is a complex multiscale issue, which is not well understood, especially at the nanoscale where the stability of the interfaces is controlled by the interplay between hydrodynamic forces and molecular forces [1-4].

The extensive literature on the droplet coalescence clearly shows that there are many different mechanisms, which depend on the characteristics of the surrounding flow [5-7]. In any case, the final stage when the droplets coalesce is controlled by molecular interactions. The hydrodynamic forces play mainly a repulsive role and are generally responsible for the droplet deformation $[2,8]$. For droplets in air, these forces are significant only at high interface approach velocity. Regarding pure fluids (without any surfactant or contaminant), the molecular forces between interfaces are attractive and there exists a threshold distance $d_{\text {min }}$ below which the droplets spontaneously form an irreversible capillary bridge among each other [9]. In this case, coalescence is a deterministic process based on the jump-to-contact instability at the air-liquid droplet interfaces. In most practical models, the coalescence time is determined by considering that the drainage process is ended when $d_{\min }$ is reached. This approach requires the determination of the $d_{\min }$ value, which is at this time unknown.

Considering van der Waals interactions ( $\mathrm{vdW}$ ), the attractive force between droplets of radii $R_{1}$ and $R_{2}$ depends on the Hamaker constant $H$ of the liquid and on the equivalent radius $R_{\text {eq }}=R_{1} R_{2} /\left(R_{1}+R_{2}\right)$. On the other hand, the surface tension $\gamma$ opposes the interface deformation. Assuming that $d_{\min }$ depends only on the three physical parameters $H, R_{\mathrm{eq}}$, and $\gamma$, dimensional analysis leads to $d_{\min } \sim R_{\mathrm{eq}}^{\alpha}(H / \gamma)^{\beta}$, with $\alpha+2 \beta=1$. By different ways, several authors have 


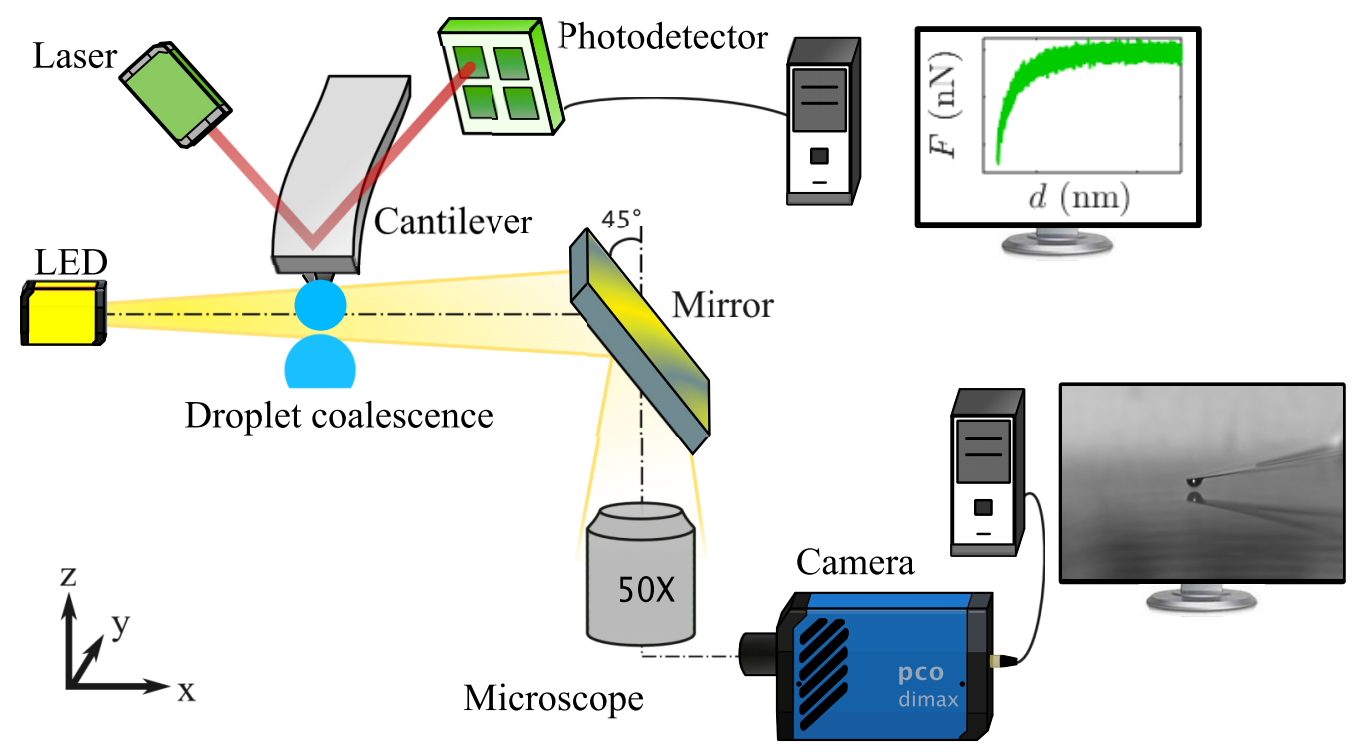

FIG. 1. Sketch of the experimental setup: AFM coupled with a high-speed camera and an inverted optical microscope.

proposed a scaling $d_{\min }=\left(H R_{\mathrm{eq}} / \gamma\right)^{1 / 3}$ [1,4], which corresponds to $\alpha=1 / 3$ and $\beta=1 / 3$. This scaling can also be written $d_{\mathrm{min}}^{*} \sim H a^{1 / 3}$ in nondimensional form, where $d_{\mathrm{min}}^{*}=d_{\min } / R_{\mathrm{eq}}$ and $H a=\frac{4 H}{3 \pi \gamma R_{\mathrm{eq}}^{2}}$. This relationship has been obtained theoretically in the case of the jump-to-contact instability of a rigid sphere approaching a liquid interface in air and considering the interface deformation prior to coalescence $[10,11]$ and confirmed experimentally by atomic force microscopy (AFM) experiments [12]. However, for coalescence between two droplets, the dependence of $d_{\text {min }}$ upon the physical parameters has not been found yet, either theoretically or experimentally.

In this Rapid Communication, we study experimentally the jump-to-contact instability between two droplets in air with the aim to measure $d_{\min }$ in situations where the attractive force is controlled by vdW interactions. The experiments are conducted by means of an AFM coupled with an inverted optical microscope and an ultrafast camera. A structural series of liquids has been considered in order to investigate the dependence of $d_{\min }$ on $H a$ over six decades. An unexpected regime is found for droplets larger than $2 \times 10^{-6} \mathrm{~m}$ for which $d_{\min }$ is independent of the droplet radii.

In order to investigate the droplet coalescence at the nanoscale, we use an AFM JPK Nanowizard 3 to experiment the jump-to-contact instability in contact mode and in frequency-modulation (FM) mode (Fig. 1). A specific cantilever holder has been built to perform coalescence experiments between droplets. It includes a $45^{\circ}$ gold mirror giving access to the side view of the droplets and a direct drive piezotransducer to vibrate the cantilever at a given amplitude and frequency. An inverted optical microscope Nikon Eclipse Ti is placed under the AFM. A 50×/0.6 long-distance objective is coupled to a high-speed PCO Dimax camera. During the experiments, the camera records the approach and coalescence of the droplets with a maximum frame rate of the camera of $1279 \mathrm{fps}$ for $2016 \times 2016$ pixels image size. Camera and AFM are synchronized via the signal access module. The camera is used to ensure an accurate positioning of the droplet fixed at the cantilever extremity just above the sessile droplet deposited on a glass substrate, to verify that no evaporation takes place during the coalescence and to provide an estimate of the instant of coalescence.

In this work, we consider a structural series of four liquids, which are characterized by a same Hamaker constant $H \simeq 6 \times 10^{-20} \mathrm{~J}[13,14]$ : diethylene glycol (2EG), triethylene glycol (3EG), tetraethylene glycol (4EG), and glycerol (Gly). The liquids are purchased from Sigma-Aldrich 


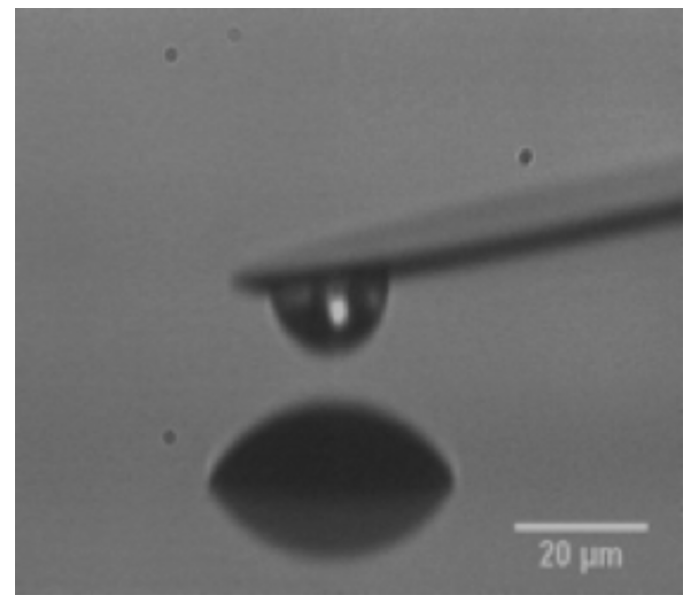

FIG. 2. Side view of a cantilever droplet before coalescence with a sessile droplet.

and are used as received after characterization using a rheometer Haake Mars III (ThermoFisher Scientific) and a tensiometer DSA100 (Krüss). Their dynamic viscosity and surface tension are $\mu=30,40,50$, and $910 \mathrm{mPa}$ and $\gamma=50,48,46$, and $63 \mathrm{mN} / \mathrm{m}$, respectively. After depositing a sessile droplet of a given liquid on a glass substrate, the droplet at the extremity of the cantilever is formed by immersing the tip in the sessile droplet. Successive immersions or redeposition on the bare substrate are performed in order to obtain pairs of droplets with different radii $R_{1}$ and $R_{2}$, where $R_{1}$ is the radius of the droplet attached to the cantilever and $R_{2}$ of the sessile droplet on the substrate (Fig. 2). Typically, we can change the radius of the cantilever droplet between 0.7 and $24 \mu \mathrm{m}$, and the radius of the sessile droplet between 4 and $74 \mu \mathrm{m}$. The droplet radii are measured on camera images just before each AFM experiment. It has been checked that the radius values of cantilever droplets measured optically are close to those calculated with the resonance frequency shift resulting from the additional mass induced by the liquid at the extremity of the cantilever.

For contact mode experiments, a Hydra 6V-200WG AppNano with a stiffness $k=0.12 \mathrm{~N} / \mathrm{m}$ and a radius $R=25 \mathrm{~nm}$ is used. For FM experiments, three different cantilevers are used: (1) PPPNCHAuD nanosensors with $k=31 \mathrm{~N} / \mathrm{m}$ and $R=12 \mathrm{~nm}$; (2) the same model with $k=31 \mathrm{~N} / \mathrm{m}$ and $R=45 \mathrm{~nm}$; and (3) PT-SiO2.Si.1 Novascan with $k=19.5 \mathrm{~N} / \mathrm{m}$ and $R=450 \mathrm{~nm}$. The cantilever stiffness is characterized by thermal noise using the deflection sensitivity derived from contact mode experiments on a silicon wafer substrate [15]. Because the stiffness of all the cantilevers is at least four times larger than the effective spring constant of the interface, which is of the order of $\gamma / 2$ [16], the jump to contact is not a consequence of a mechanical instability of the cantilever, but is the result of a hydrodynamic instability of the droplet interfaces. In contact and FM modes, the force $F$ and the frequency shift $\Delta f$, respectively, are monitored versus the actual distance $d$ between the interfaces of the droplets. The latter is calculated by the following relationship: $d=z+\delta-\delta_{m}+d_{\min }$, where $z$ is the piezoelevation whose origin is arbitrarily taken at the jump to contact (detected by the maximum value of the gradient of the experimental curves), $\delta$ is the cantilever deflection measured at the distance $z$, and $\delta_{m}$ is the cantilever deflection measured at $z=0$.

In order to determine $d_{\min }$, the force curves are fitted by

$$
F=\frac{H R_{\mathrm{eq}}}{6 d^{2}},
$$

corresponding to the interaction between two rigid spheres separated by a distance $d$. The assumption that the droplets remain spherical up to the jump-to-contact instability $\left(d \geqslant d_{\min }\right)$ is valid because (1) the interface deformation prior to the jump to contact is small compared to the 


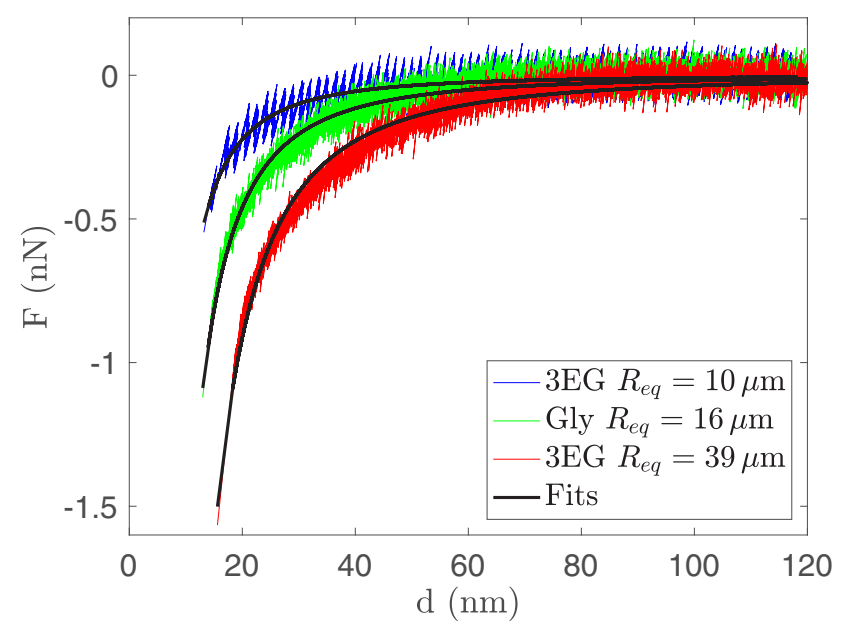

FIG. 3. Contact AFM experiments: force versus displacement. Fits are obtained by means of Eq. (1).

droplet radii [12] and (2) the viscous effects in the interstitial film are negligible according to the criterion: $\frac{V \eta R_{\mathrm{eq}}^{2}}{\gamma d_{\min }^{2}} \ll 1$, where $V$ and $\eta$ are the droplet approach velocity and the air viscosity.

In FM mode, the shift frequency curve is fitted by the following relationship derived and validated in [12]:

$$
\frac{\Delta f}{f_{0}}=-\frac{1}{2 \pi k A^{2}} \int_{0}^{2 \pi} F \times A \cos (x) d x,
$$

where $f_{0}$ is the cantilever eigenfrequency and $A$ is the oscillation amplitude. In both contact and FM modes, the fits are done by least-square method where $d_{\min }$ and $R_{\mathrm{eq}}$ are the free parameters for minimization. The values of $R_{\mathrm{eq}}$ are consistent with optical measurements, which makes us confident with the values of $d_{\min }$ provided by this method.

Examples of measured force and frequency shift curves together with the fits are presented in Figs. 3 and 4, respectively. These curves show the increase of the absolute value of the attractive force between the two droplets or that of the frequency shift as the interface distance decreases. As expected, the vdW interaction range expands as $R_{\text {eq }}$ becomes larger. We observe a very good agreement between the experimental curves and Eqs. (1) and (2), which confirms that the droplets do not deform significantly up to $d_{\min }$. Even if they are more difficult to execute, FM experiments provide more accurate data and the experimental frequency shift curves are less noisy. This is consistent with the fact that in dynamic mode, at resonance, the sensitivity is increased by $Q$ the quality factor of the cantilever which, in our case, is of the order of 100 . However, both AFM modes lead to similar results, which validates the determination methods of $d_{\min }$. In the following, the results of the two AFM modes will not be distinguished. Figure 5 displays the measured values of $d_{\text {min }}$ as a function of the radius $R_{\text {eq }}$ for a series of droplets of different sizes and liquids. It is important to note that our droplet manipulation procedure allows one to probe a wide range of values of $R_{\text {eq }}$ spanning over three decades while varying the ratio of radii $R_{1} / R_{2}$ from 0.04 to 1 . We observe that, for small values of $R_{\text {eq }}$, as expected, $d_{\text {min }}$ increases with $R_{\text {eq }}$ following a power law which is compatible with a $R_{\mathrm{eq}}^{1 / 3}$ dependence. Interestingly, for $R_{\mathrm{eq}} \geqslant 2 \mu \mathrm{m}, d_{\text {min }}$ does not depend anymore on $R_{\text {eq }}$ and reaches a constant value $d_{\min } \approx 15 \mathrm{~nm}$. Moreover, it turns out that $d_{\text {min }}$ is independent of $R_{1} / R_{2}$ over the whole range of $R_{\text {eq }}$ investigated. This is evidenced in Fig. 5 where the data points are colored depending on the $R_{1} / R_{2}$ value (blue points for $R_{1} / R_{2}<0.3$ and red points for $R_{1} / R_{2}>0.3$ ). We observe that the two series of data follow the same trend with the two regimes. Hence, similar values of $d_{\min }$ can be obtained with similar values of $R_{\text {eq }}$ but different 


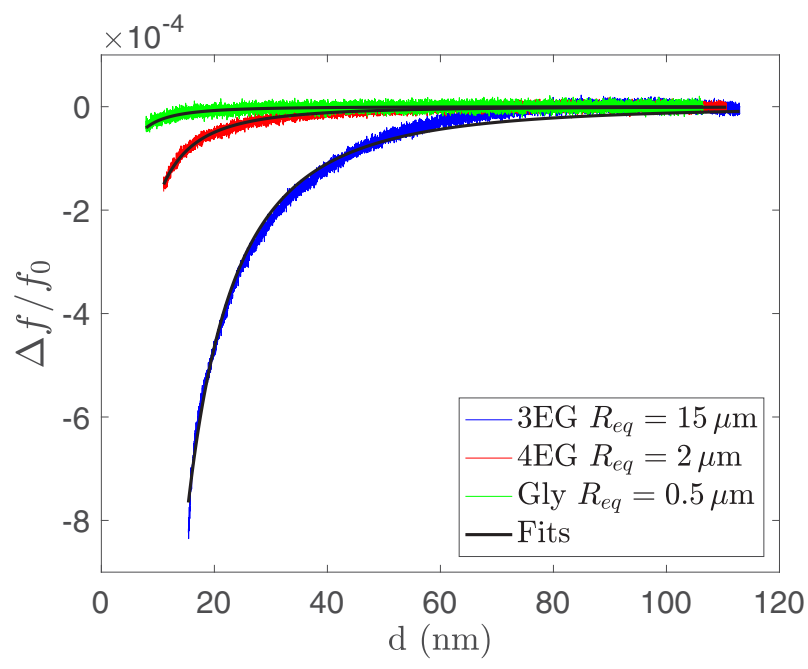

FIG. 4. FM-AFM experiments: frequency shift versus displacement. Fits are obtained by means of Eqs. (1) and (2).

$R_{1} / R_{2}$ ratios. In order to interpret these results, $d_{\text {min }}^{*}$ is plotted as a function of $H a$ in Fig. 6 . This nondimensional form allows one to get rid of the nature of liquids and to obtain a master curve including all experimental data. The blue and red dots represent the data of Fig. 5: the regime where $d_{\min }$ is constant corresponds to the new power law $d_{\min }^{*} \propto H a^{1 / 2}$, while the regime where $d_{\min }$ increases corresponds to the already known power law $d_{\min }^{*} \propto H a^{1 / 3}$. The black dots represent the results of a previous AFM study of the jump to contact between a puddle and a droplet or a wetted tip [12], performed with the same series of liquids. In these experiments, $R_{\mathrm{eq}}=R_{1}$ (because $R_{2}$ is infinite) and the definition of $\mathrm{Ha}$ is identical. At variance with the present study, this previous work thus dealt with only a single radius ratio $\left(R_{1} / R_{2} \approx 0\right)$. Furthermore, it investigated a different range of radius, which did not include large enough droplets to clearly identify the $\mathrm{Ha}^{1 / 2}$ regime. As a consequence, even though this regime can now be discerned in Fig. 6 for $\mathrm{Ha} \leqslant 10^{-7}$, the

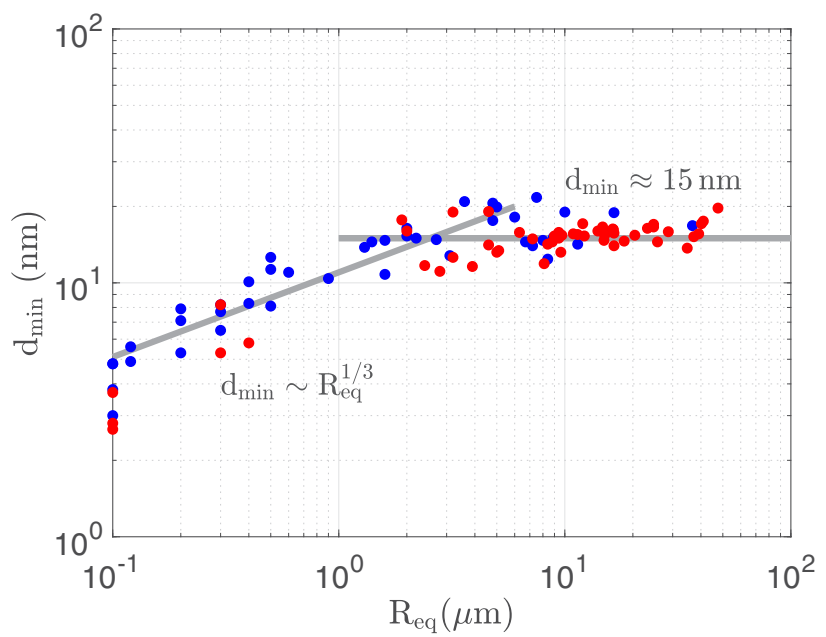

FIG. 5. $d_{\text {min }}$ versus $R_{\text {eq }}$. The blue dots correspond to $R_{1} / R_{2}<0.3$ and the red dots to $R_{1} / R_{2}>0.3$. 


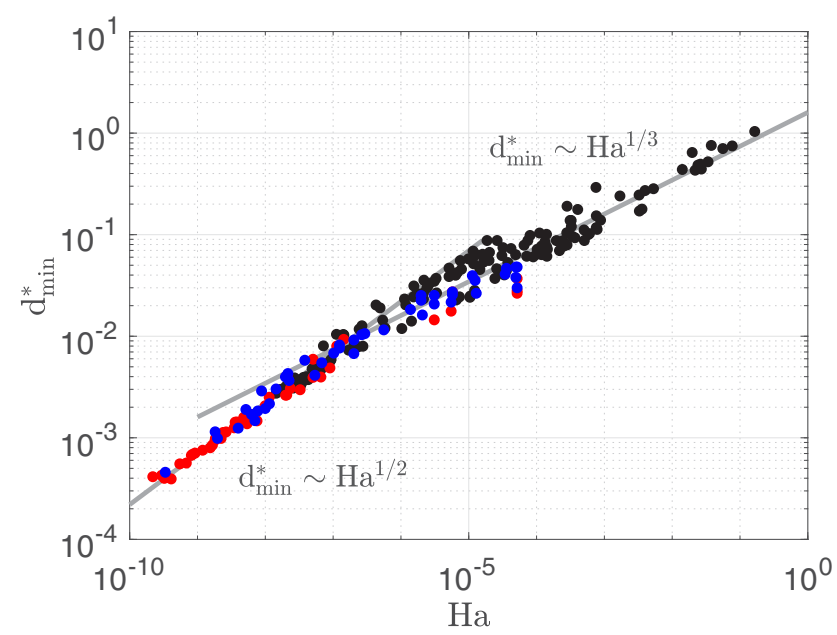

FIG. 6. $d_{\mathrm{min}}^{*}$ versus $\mathrm{Ha}$. The blue dots represent the data of the jump to contact between droplets. The blue dots correspond to $R_{1} / R_{2}<0.3$ and the red dots to $R_{1} / R_{2}>0.3$. The black dots represent the data from [12] of the jump to contact between a liquid puddle and a droplet, which corresponds to $R_{1} / R_{2} \approx 0$.

transition between the two regimes was not noticed in [12] where only the $\mathrm{Ha}^{1 / 3}$ regime was pointed out. It is remarkable that the results of the two studies which span over nine orders of magnitude in $H a$ exactly show the same trends, which confirms that $d_{\text {min }}^{*}$ depends only on $H a$ and not on radius ratio for $R_{1} / R_{2}$ ranging from 0 to 0.9 . The combination of these two types of experiments thus allows us to clearly identify two regimes which can be approximated by $d_{\min }^{*}=22 \mathrm{Ha}^{1 / 2}$ for $H a \lesssim 1.5 \times 10^{-7}$ and $d_{\min }^{*}=1.6 H a^{1 / 3}$ for larger $H a$. While the regime for the small droplets in $\mathrm{Ha}^{1 / 3}$ involves that the characteristic scale of $d_{\min }$ is $\left(H R_{\mathrm{eq}} / \gamma\right)^{1 / 3}$, as already established by several authors $[1,9,11,12]$, the regime in $H a^{1 / 2}$ for large droplets exhibits a characteristic length $(H / \gamma)^{1 / 2}$. This regime can be interpreted by considering that $d_{\min }$ becomes independent of $R_{\text {eq }}$ for large enough droplets. In this case, the dimensional analysis mentioned above, leads to $\alpha=0$ and $\beta=1 / 2$ and therefore $d_{\min } \sim(H / \gamma)^{1 / 2}$. However, this new behavior is observed from a value as small as $2 \mu \mathrm{m}$, both for droplet-droplet coalescence and droplet-puddle coalescence. This result is crucial for understanding and modeling of coalescence. Hence, except for very specific situations involving submicrometer droplets, the jump to contact responsible for coalescence occurs at a value of $d_{\min }$ of the order of $10 \mathrm{~nm}$, which depends only on the physical parameters $H$ and $\gamma$, and not on droplet size.

In this Rapid Communication we reported on an experimental study of the jump-to-contact instability in air. Using an AFM setup and a dedicated protocol to manipulate micron-size droplets, we provide a large set of experimental data on the distance $d_{\min }$ at which coalescence occurs. While for small droplets we confirm the theoretical estimations $d_{\min }^{*} \sim \mathrm{Ha}^{1 / 3}$ generally accepted, an unexpected regime $d_{\min }^{*} \sim H a^{1 / 2}$ appears for droplets of sizes larger than $2 \mu \mathrm{m}$ which may modify the description of coalescence. The conclusions of this work apply for all situations where hydrodynamic forces within the interstitial film between droplets are negligible and molecular forces are described by vdW interactions. Future work should now address the coalescence of droplets immersed in liquid. First, the hydrodynamic forces will cause the interface deformation and delay or prevent the reaching of $d_{\min }$. Second, electrical charges may screen the vdW forces and possibly decrease the $d_{\min }$ value. An experimental strategy similar to the one developed in this work that allows us to reveal the regimes of droplet coalescence in air should be fruitful as well in liquids.

The authors would like to thank C. Mortagne and M. Ogier for their help in the AFM experiments and the FERMaT Federation for its support in instrumentation. 
[1] A. K. Chesters, The modelling of coalescence processes in fluid-liquid dispersions: A review of current understanding, Chem. Eng. Res. Des. 69(A4), 259 (1991).

[2] J. Eggers, J. R. Lister, and H. A. Stone, Coalescence of liquid drops, J. Fluid Mech. 401, 293 (1999).

[3] A. Frohn and N. Roth, Droplet systems, in Dynamics of droplets (Springer, Berlin, Heidelberg, 2000).

[4] N. Chen, T. Kuhl, R. Tadmor, Q. Lin, and J. Israelachvili, Large Deformations during the Coalescence of Fluid Interfaces, Phys. Rev. Lett. 92, 024501 (2004).

[5] Y. Liao and D. Lucas, A literature review on mechanisms and models for the coalescence process of fluid particles, Chem. Eng. Sci. 65, 2851 (2010).

[6] D. Y. C. Chan, E. Klaseboer, and R. Manica, Film drainage and coalescence between deformable drops and bubbles, Soft Matter 7, 2235 (2011).

[7] J. E. Sprittles and Y. D. Shikhmurzaev, Coalescence of liquid drops: Different models versus experiment, Phys. Fluids 24, 122105 (2012).

[8] H. J. Lockie, R. Manica, G. W. Stevens, F. Grieser, D. Y. C. Chan, and R. R. Dagastine, Precision AFM measurements of dynamic interactions between deformable drops in aqueous surfactant and surfactant-free solutions, Langmuir 27, 2676 (2011).

[9] R. Ledesma-Alonso, D. Legendre, and P. Tordjeman, Nanoscale Deformation of a Liquid Surface, Phys. Rev. Lett. 108, 106104 (2012).

[10] R. Ledesma-Alonso, P. Tordjeman, and D. Legendre, Multiscale deformation of a liquid surface in interaction with a nanoprobe, Phys. Rev. E 85, 061602 (2012).

[11] D. B. Quinn, J. Feng, and H. A. Stone, Analytical model for the deformation of a fluid-fluid interface beneath an AFM probe, Langmuir 29, 1427 (2013).

[12] C. Mortagne, V. Chireux, R. Ledesma-Alonso, M. Ogier, F. Risso, T. Ondarçuhu, D. Legendre, and $\mathrm{Ph}$. Tordjeman, Near-field deformation of a liquid interface by atomic force microscopy, Phys. Rev. E 96, 012802 (2017).

[13] J. Visser, On Hamaker constants: A comparison between Hamaker constants and Lifshitz-van der Waals constants, Adv. Colloid Interface Sci. 3, 331 (1972).

[14] J. N. Israelachvili, Intermolecular and Surface Forces (Academic, New York, 2011).

[15] N. A. Burnham, X. Chen, C. S. Hodges, G. A. Matei, E. J. Thoreson, C. J. Roberts, M. C. Davies, and S. J. B. Tendler, Comparison of calibration methods for atomic-force microscopy cantilevers, Nanotechnology 14, 1 (2003).

[16] J. Dupré de Baubigny, M. Benzaquen, L. Fabié, M. Delmas, J.-P. Aimé, M. Legros, and T. Ondarçuhu, Shape and effective spring constant of liquid interfaces probed at the nanometer scale: Finite size effects, Langmuir 31, 9790 (2015). 